Gut and Liver, Vol. 11, No. 3, May 2017, pp. 409-416

\title{
Predictive Factors for Complete Response and Recurrence after Transarterial Chemoembolization in Hepatocellular Carcinoma
}

Shin Ok Jeong ${ }^{1}$, Eui Bae Kim ${ }^{1}$, Soung Won Jeong ${ }^{1}$, Jae Young Jang ${ }^{1}$, Sae Hwan Lee ${ }^{2}$, Sang Gyune Kim ${ }^{3}$, Sang Woo Cha ${ }^{1}$, Young Seok Kim ${ }^{3}$, Young Deok Cho ${ }^{1}$, Hong Soo Kim², Boo Sung Kim ${ }^{1}$, Yong Jae Kim ${ }^{4}$, Dong Erk Goo ${ }^{4}$, and Su Yeon Park

${ }^{1}$ Institute for Digestive Research and Digestive Disease Center, Department of Internal Medicine, Soonchunhyang University Hospital, Seoul, ${ }^{2}$ Department of Internal Medicine, Soonchunhyang University Hospital, Cheonan, ${ }^{3}$ Department of Internal Medicine, Soonchunhyang University Hospital, Bucheon, Departments of ${ }^{4}$ Radiology and ${ }^{5}$ Biostatistics, Soonchunhyang University Hospital, Seoul, Korea

Background/Aims: To investigate the predictive factors for complete response (CR) and recurrence after $\mathrm{CR}$ in patients with hepatocellular carcinoma (HCC) treated with transarterial chemoembolization (TACE). Methods: Among 691 newly diagnosed HCC patients, 287 were treated with TACE as a first therapy. We analyzed the predictive factors for $\mathrm{CR}$, recurrence after $\mathrm{CR}$, and overall survival (OS). Results: Eighty-one patients (28.2\%) achieved CR after TACE, and recurrence after CR was detected in 35 patients (43.2\%). In multivariate analyses, tumor size $(\leq 5 \mathrm{~cm})$ and single nodularity were predictive factors for $\mathrm{CR}$, with hazard ratios (HRs) of 0.35 $(p=0.002)$ and $0.41(p<0.001)$, respectively. Elevated serum $\alpha$-fetoprotein (AFP) (>20 ng/mL) level and multinodularity exhibited significant relationships with recurrence after $\mathrm{CR}$, with HRs of $2.220(p=0.026)$ and $3.887(p<0.001)$, respectively. Tumor size $(>5 \mathrm{~cm})$, multinodularity, elevated serum AFP $(>20$ $\mathrm{ng} / \mathrm{mL}$ ) level, Child-Turcotte-Pugh score (B and C), and portal vein thrombosis were significant factors for OS. Conclusions: In patients treated with TACE as a first therapy, tumor size $(\leq 5$ $\mathrm{cm}$ ) and single nodularity were predictive factors for $\mathrm{CR}$, and multinodularity and elevated serum AFP (>20 ng/mL) levels were predictive factors for recurrence after CR. These factors were also significant for OS. (Gut Liver 2017;11:409-416)

Key Words: Carcinoma, hepatocellular; Chemoembolization, therapeutic; Complete response; Recurrence

\section{INTRODUCTION}

Hepatocellular carcinoma (HCC) is the sixth most common tumor worldwide and the third leading cause of tumor-related mortality, exceeded only by tumors of the lung and stomach. ${ }^{1,2}$ It is more prevalent in sub-Saharan Africa and Central and Southeast Asia. HCC is a major and often therapeutically frustrating interdisciplinary problem with a rising incidence in Western countries due to increasing prevalence of risk factors such as diabetes, hepatitis $\mathrm{C}$ virus, and excessive alcohol intake. ${ }^{3}$

The prognosis of HCC patients remains poor due to the advanced stage of cancer at diagnosis and high recurrence rates of nearly $80 \%$ at 5 years after resection. ${ }^{4}$ Resection and transplantation, in fact, achieve 5-year survival rates of 50\% or higher ${ }^{5}$ and should be considered as the first treatment option in this setting. However, curative therapy options such as resection, liver transplantation and local ablation therapies are only applicable during the early stages of the disease. ${ }^{6-8}$ Unfortunately, $60 \%$ to $70 \%$ of patients with HCC are diagnosed by the time the disease reaches an advanced stage, ${ }^{9}$ and thus most patients are treated in palliative situations. Transarterial chemoembolization (TACE) is the best therapeutic option in this situation.

HCC patients treated with palliative TACE showed survival rates of $82 \%, 47 \%$, and $26 \%$ at $1-, 3-$, and 5-year, respectively. ${ }^{10}$ The survival rate after palliative TACE was influenced most by good performance status, complete response (CR) and lower bilirubin levels. ${ }^{11}$ TACE could be another option when curative treatments such as surgery or radiofrequency ablation (RFA) are unsuccessful or unfeasible. TACE used during early-stage HCC resulted in 1-, 3-, and 5-year overall survival (OS) rates of 91\%, $66 \%$, and 52\%, respectively. Serum albumin, tumor size, tumor number and recurrence interval influenced mortality. ${ }^{12}$

The risk factors for recurrence after TACE in patients with

Correspondence to: Soung Won Jeong

Institute for Digestive Research and Digestive Disease Center, Department of Internal Medicine, Soonchunhyang University Hospital, 59 Daesagwanro, Yongsan-gu, Seoul 04401, Korea

Tel: +82-2-709-3075, Fax: +82-2-709-9696, E-mail: jeongsw@schmc.ac.kr

Received on December 31, 2015. Revised on August 4, 2016. Accepted on September 1, 2016. Published online February 17, 2017

pISSN 1976-2283 eISSN 2005-1212 https://doi.org/10.5009/gnl16001

Shin Ok Jeong and Eui Bae Kim contributed equally to this work as first authors.

(a) This is an Open Access article distributed under the terms of the Creative Commons Attribution Non-Commercial License (http://creativecommons.org/licenses/by-nc/4.0) which permits unrestricted non-commercial use, distribution, and reproduction in any medium, provided the original work is properly cited. 
HCC have been reported to include location of HCC, size of HCC, tumor markers, viral markers, number of HCCs, age, and liver function. ${ }^{13-18}$

However, the predictive factors for $\mathrm{CR}$, recurrence after $\mathrm{CR}$, and their association to OS has not yet been elucidated. Here, we investigated the predictive factors for CR, recurrence after $\mathrm{CR}$, and their association to OS in patients with HCC treated with TACE.

\section{MATERIALS AND METHODS}

\section{Patients}

Six hundred ninety-one newly diagnosed HCC patients admitted to Soonchunhyang University Hospital, Seoul, Korea, between January 2004 and December 2014 were investigated retrospectively. Of these 691 patients, excluding the patients who were treated with other treatment option as a first therapy or the patients who were not treated or the patients who were lost to follow-up, 287 consecutive HCC patients underwent TACE as a first treatment were evaluated. The exclusion criteria for TACE were (1) surgical candidates; (2) presenting major portal vein thrombosis; (3) hepatic encephalopathy; (4) presenting a current infection; (5) recent episode of gastrointestinal bleeding within the past month; and (6) uncontrolled ascites. TACE was also performed for early-stage HCC (stages I and II) if the patients were not suitable for resection, RFA or liver transplantation because of poor liver function or a location difficult to access by surgery or RFA, or difficulty obtaining a donor liver. This study was carried out according to the principles of the Declaration of Helsinki 1964 and was approved by the Ethics Review Boards of Soonchunhyang University Seoul Hospital.

\section{Diagnosis}

HCC diagnosis was based on histological criteria and/or imaging techniques, as proposed by the American Association for the Study of Liver Diseases. ${ }^{19}$ For the diagnosis of HCC, nodules found on ultrasound surveillance that are smaller than $1 \mathrm{~cm}$ should be followed up with ultrasound at 3-month intervals. Lesions larger than $1 \mathrm{~cm}$ in diameter should be evaluated by dynamic magnetic resonance imaging or helical multidetector computed tomography (CT) scan using contrast. If the appearance is typical for HCC, no further investigation is required. Tumors were staged according to the modified Union Internationale Contre le Cancer (modified UICC) classification system. ${ }^{20}$ Early stage was defined as stages I and II, and advanced stage was restricted to stages III and IV. CR was defined as the disappearance of any intratumoral arterial enhancement in all target lesions or compact lipiodol uptake after TACE according to modified Response Evaluation Criteria in Solid Tumours. ${ }^{21}$ Tumor recurrence was determined when a new lesion appeared or when an enhanced portion was seen within or at the margin of the original mass on the next follow-up CT scan after initial
$\mathrm{CR}$. The recurrence pattern was classified as local recurrence ( $\leq 2 \mathrm{~cm}$ from the primary tumor) or distant recurrence $(>2 \mathrm{~cm}$ apart from the primary tumor) according to the location of the recurred tumor.

\section{Treatment}

All TACE procedures were performed by two experienced interventional radiologists. Hepatic angiography was performed using angiographic catheters, followed by selection of segmental arterial feeders. An iodized oil-doxorubicin hydrochloride emulsion was then administered into the feeders. Once the flow became sluggish, gelatin sponge particles mixed with mitomycin- $\mathrm{C}$ and contrast material were administered into the feeders until blood flow stopped completely. TACE was withheld or discontinued whenever vascular contraindications, poor hepatic function, severe adverse effects or progressive disease developed. TACE was performed at baseline and 4 weeks; after every TACE, a multiphasic CT scan was performed. When CT scan after TACE detected viable tumors, repeat TACE was performed immediately. If viable tumors were not detected on CT scan, additional CT scans were conducted at 2-month intervals. The routine post-TACE follow-up protocol included biochemical liver function tests and serum $\alpha$-fetoprotein (AFP) level measurements.

\section{Predictive factors}

The baseline characteristics of 287 patients treated with TACE were evaluated. We analyzed the clinical and biological factors that influenced initial CR using the following parameters: age, gender, viral markers (hepatitis B virus surface antigen and hepatitis $C$ virus antibody), liver cirrhosis, model for end-stage liver disease (MELD) score, Child-Pugh score, serum AFP, size of tumors, number of tumors, portal vein thrombosis. In addition, we analyzed the risk factors for recurrence after TACE-induced $\mathrm{CR}$ and OS using the same parameters.

\section{Statistical analysis}

Univariate analysis was computed using the log-rank test to analyze differences between groups. Variables that were not statistically significant at univariate analysis ( $p>0.05)$ were excluded from the multivariate analysis. The Cox proportional hazard model was used for multivariate analysis, and stepwise selection was performed to identify the best subset of predictors. The cumulative recurrence rates were calculated from the time of diagnosis of HCC using the Kaplan-Meier method. Data were analyzed using commercially available statistical software packages SPSS version 19.0 (SPSS Inc., Chicago, IL, USA). All reported $p$-values are two-sided, with $\mathrm{p}<0.05$ considered statistically significant. 


\section{RESULTS}

\section{Patients}

Of 287 patients treated with TACE, 81 patients achieved CR and recurrent HCC after CR was detected in 35 patients. The flow chart of patients is summarized in Fig. 1. The baseline characteristics of 287 HCC patients treated with TACE are summarized in Table 1. The median and mean of each characteristic were determined according to the results of the histogram. The mean age of the 287 patients was $60.71 \pm 11.08$ years. The patients included 208 males (72.5\%) and 79 females (27.5\%). With respect to the modified UICC stage, 118 patients were early stage (41.1\%) and 169 advanced stage (58.9\%). One hundred sixty-five patients had an AFP level $>20 \mathrm{ng} / \mathrm{mL}$. The median maximal diameter of the tumors was $3.2 \mathrm{~cm}$ (range, 0.9 to 20.0 $\mathrm{cm})$. Forty-six patients (16\%) had portal vein thrombosis.

\section{Complete response}

Among 287 HCC patients, eighty-one patients (28.2\%) with CR after TACE were followed for a median period of 34 months (range, 5 to 136 months). The patients had a mean age of $59.3 \pm 12.18$ years. The patients included 53 males $(65.4 \%)$ and 28 females (34.6\%). Fifty-one patients (63\%) were infected with hepatitis B virus and 16 (19.8\%) with hepatitis C virus.

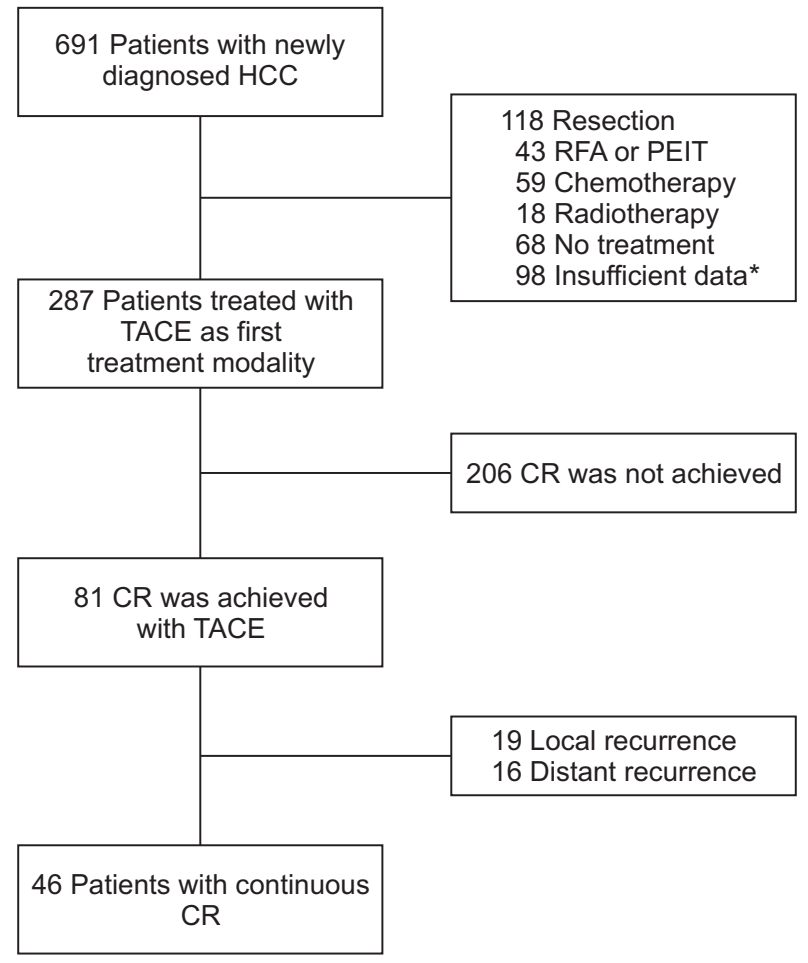

Fig. 1. Flowchart of patients.

HCC, hepatocellular carcinoma; RFA, radiofrequency ablation; PEIT, percutaneous ethanol injection therapy; TACE, transarterial chemoembolization; CR, complete response.

*Insufficient data, transfer to another hospital or loss to follow-up.
The mean albumin level was $3.53 \pm 0.71 \mathrm{~g} / \mathrm{dL}$. The median total bilirubin, prothrombin time and MELD were $0.9 \mathrm{mg} / \mathrm{dL}$ (range, 0.3 to $4.5 \mathrm{mg} / \mathrm{dL}$ ), $81 \%$ (range, $32.3 \%$ to $100 \%$ ) and 6.09 (range, 2.46 to 15.20 ), respectively. With respect to the modified UICC stage, 47 patients had early stage (58\%) and 34 advanced stage (42\%). Regarding the Child-Pugh score, 54 patients were class A (66.7\%), 22 were class B (27.1\%), and five were class C (6.2\%). Thirty-six patients had elevated serum AFP (>20 ng/mL) level. The median maximal diameter of the tumors was $2.2 \mathrm{~cm}$ (range, 1 to $9.8 \mathrm{~cm})$. Twenty-nine (35.8\%) had a multinodular pattern. Four patients had portal vein thrombosis (4.9\%).

\section{Predictive factors for $\mathrm{CR}$}

Elevated serum AFP ( $>20 \mathrm{ng} / \mathrm{mL}$ ) level (hazard ratio [HR], 1.709; 95\% confidence interval [CI], 1.101 to 2.650; $\mathrm{p}=0.017)$, tumor size $(>5 \mathrm{~cm})$ (HR, 3.441;95\% CI, 1.775 to 6.673 ; $\mathrm{p}<0.001$ ), multinodularity (HR, 2.658; 95\% CI, 1.687 to 4.187 ; $\mathrm{p}<0.001)$ and portal vein thrombosis $(\mathrm{HR}, 3.562$; 95\% CI, 1.303 to $9.739 ; p=0.013$ ) were correlated with $\mathrm{CR}$ after TACE in univariate analysis. On multivariate analysis, tumor size $(>5 \mathrm{~cm})$ (HR, 2.840; 95\% CI, 1.449 to 5.565; $\mathrm{p}=0.002$ ) and multinodularity (HR, 2.435; 95\% CI, 1.535 to $3.861 ; \mathrm{p}<0.001$ ) were significantly correlated with CR after TACE (Table 2). When we change multinodularity to single nodularity, HR was 0.35 (95\% CI, 0.180

Table 1. Baseline Characteristics of Patients with Transarterial Chemoembolization $(\mathrm{n}=287)$

\begin{tabular}{lc}
\hline \multicolumn{1}{c}{ Characteristic } & Value \\
\hline Age, yr & $60.71 \pm 11.08$ \\
Male sex & $208(72.5)$ \\
Aetiology & \\
$\quad$ HBV & $172(59.9)$ \\
HCV & $45(15.7)$ \\
$\quad$ NBNC & $70(24.4)$ \\
Albumin, g/dL & $3.49 \pm 0.69$ \\
Total bilirubin, mg/dL & $1.0(0.2-13.9)$ \\
Prothrombin time (\%) & $84(23-100)$ \\
MELD score & $6.25(2.19-15.20)$ \\
Tumor stage (I, II/III, IV) & $118 / 169(41.1 / 58.9)$ \\
Liver cirrhosis & $220(76.7)$ \\
CTP score (A/B/C) & $192 / 80 / 15(66.9 / 27.9 / 5.2)$ \\
AFP (>20 ng/mL) & $165(57.5)$ \\
Tumor size (>5 cm) & $92(32.1)$ \\
Nodularity (multinodular) & $165(57.5)$ \\
TACE session (times) for CR $(1 / 2 / \geq 3)$ & $60 / 15 / 6$ \\
Portal vein thrombosis & $46(16)$ \\
\hline
\end{tabular}

Data are presented as mean \pm SD, number (\%), or median (range).

$\mathrm{HBV}$, hepatitis B virus; HCV, hepatitis C virus; NBNC, nonhepatitis $\mathrm{B}$ and nonhepatitis C; MELD, model for end-stage liver disease; CTP, Child-Turcotte-Pugh; AFP, $\alpha$-fetoprotein; TACE, transarterial chemoembolization; CR, complete response. 
Table 2. Predictive Factors for Complete Response

\begin{tabular}{|c|c|c|c|c|c|c|}
\hline \multirow{2}{*}{ Variable } & \multicolumn{3}{|c|}{ Univariate analysis } & \multicolumn{3}{|c|}{ Multivariate analysis } \\
\hline & $\mathrm{HR}$ & $95 \% \mathrm{Cl}$ & $\mathrm{p}$-value & HR & $95 \% \mathrm{Cl}$ & $\mathrm{p}$-value \\
\hline Age (>60 yr) & 0.805 & $0.520-1.245$ & 0.330 & & & \\
\hline Male sex & 1.404 & $0.888-2.221$ & 0.146 & & & \\
\hline $\mathrm{HBV}$ & 1.171 & $0.668-2.053$ & 0.582 & & & \\
\hline $\mathrm{HCV}$ & 0.655 & $0.363-1.183$ & 0.161 & & & \\
\hline Liver cirrhosis & 1.759 & $0.953-3.248$ & 0.071 & & & \\
\hline MELD score $(>10)$ & 0.931 & $0.429-2.022$ & 0.857 & & & \\
\hline CTP score $(\mathrm{A} / \mathrm{B}, \mathrm{C})$ & 1.057 & $0.666-1.678$ & 0.815 & & & \\
\hline AFP (>20 ng/mL) & 1.709 & $1.101-2.650$ & 0.017 & & & \\
\hline Tumor size $(>5 \mathrm{~cm})$ & 3.441 & $1.775-6.673$ & $<0.001$ & 2.840 & $1.449-5.565$ & 0.002 \\
\hline Nodularity (multinodular) & 2.658 & $1.687-4.187$ & $<0.001$ & 2.435 & $1.535-3.861$ & $<0.001$ \\
\hline Portal vein thrombosis & 3.562 & $1.303-9.739$ & 0.013 & & & \\
\hline
\end{tabular}

HR, hazard ratio; CI, confidence interval; HBV, hepatitis B virus; HCV, hepatitis C virus; MELD, model for end-stage liver disease; CTP, ChildTurcotte-Pugh; AFP, $\alpha$-fetoprotein.

Table 3. Predictive Factors for Recurrence

\begin{tabular}{|c|c|c|c|c|c|c|}
\hline \multirow{2}{*}{ Variable } & \multicolumn{3}{|c|}{ Univariate analysis } & \multicolumn{3}{|c|}{ Multivariate analysis } \\
\hline & $\mathrm{HR}$ & $95 \% \mathrm{CI}$ & p-value & $\mathrm{HR}$ & $95 \% \mathrm{CI}$ & $\mathrm{p}$-value \\
\hline Age (>60 yr) & 0.595 & $0.290-1.222$ & 0.157 & & & \\
\hline Male sex & 0.502 & $0.234-1.073$ & 0.075 & & & \\
\hline $\mathrm{HBV}$ & 2.053 & $0.712-5.922$ & 0.183 & & & \\
\hline $\mathrm{HCV}$ & 2.328 & $0.680-7.969$ & 0.178 & & & \\
\hline Liver cirrhosis & 1.188 & $0.458-3.083$ & 0.723 & & & \\
\hline MELD score $(>10)$ & 0.556 & $0.133-2.326$ & 0.422 & & & \\
\hline CTP score $(\mathrm{A} / \mathrm{B}, \mathrm{C})$ & 1.127 & $0.551-2.306$ & 0.743 & & & \\
\hline $\operatorname{AFP}(>20 \mathrm{ng} / \mathrm{mL})$ & 2.544 & $1.285-5.036$ & 0.007 & 2.220 & $1.098-4.488$ & 0.026 \\
\hline Tumor size $(>5 \mathrm{~cm})$ & 1.167 & $0.452-3.015$ & 0.750 & & & \\
\hline Nodularity (multinodular) & 5.487 & $2.725-11.047$ & $<0.001$ & 5.143 & $2.532-10.444$ & $<0.001$ \\
\hline Portal vein thrombosis & 1.335 & $0.319-5.588$ & 0.693 & & & \\
\hline
\end{tabular}

HR, hazard ratio; CI, confidence interval; HBV, hepatitis B virus; HCV, hepatitis C virus; MELD, model for end-stage liver disease; CTP, ChildTurcotte-Pugh; AFP, $\alpha$-fetoprotein.

to $0.690 ; \mathrm{p}=0.002)$. Among the 81 patients with $\mathrm{CR}, 71$ patients had tumor size $(\leq 5 \mathrm{~cm})$, and 10 patients $(12.3 \%)$ had tumor size $(>5 \mathrm{~cm})$. Fifty-two patients $(64.2 \%)$ had single nodularity and 29 patients (35.8\%) showed multinodularity.

\section{Recurrence}

Of the 81 patients with $\mathrm{CR}$, recurrent HCC was detected in 35 patients (43.2\%). The median follow-up period to recurrence after achieving $\mathrm{CR}$ was 12 months (range, 2 to 67 months). The overall 1-, 2-, and 3-year cumulative recurrence rates were 23.1\%, 43.7\%, and 45.8\%, respectively. There were 19 local recurrences (54.3\%) and 16 distant recurrence (45.7\%). The overall 1-, 2-, and 3-year cumulative local recurrence rates were 15.5\%, $26 \%$, and 26\%, respectively. The overall 1-, 2-, and 3-year cumulative distant recurrence rates were 8.9\%, 23.6\%, and 26.5\%, respectively.

\section{Predictive factors for recurrence}

In univariate analysis, multinodularity $(\mathrm{HR}, 5.487 ; 95 \% \mathrm{CI}$, 2.725 to $11.047 ; \mathrm{p}<0.001$ ) and elevated serum AFP (>20 ng/ $\mathrm{mL}$ ) level (HR, 2.544; 95\% CI, 1.285 to 5.036; $\mathrm{p}=0.007$ ) were associated with HCC recurrence after TACE-induced CR. In multivariate analysis, multinodularity (HR, 5.143; 95\% CI, 2.532 to 10.444; $\mathrm{p}<0.001$ ) and elevated serum AFP (>20 ng/mL) level (HR, 2.220; 95\% CI, 1.098 to $4.488 ; p=0.026$ ) were also associated with HCC recurrence after TACE-induced CR (Table 3). Of the 81 patients with $\mathrm{CR}, 29$ patients (35.8\%) has multinodularity, and 36 patients (44.4\%) has elevated serum AFP (>20 ng/mL) level.

The recurrence rate of multinodularity (22/29 patients) was higher than that of single nodularity (13/52 patients) (75.9\% vs 
A

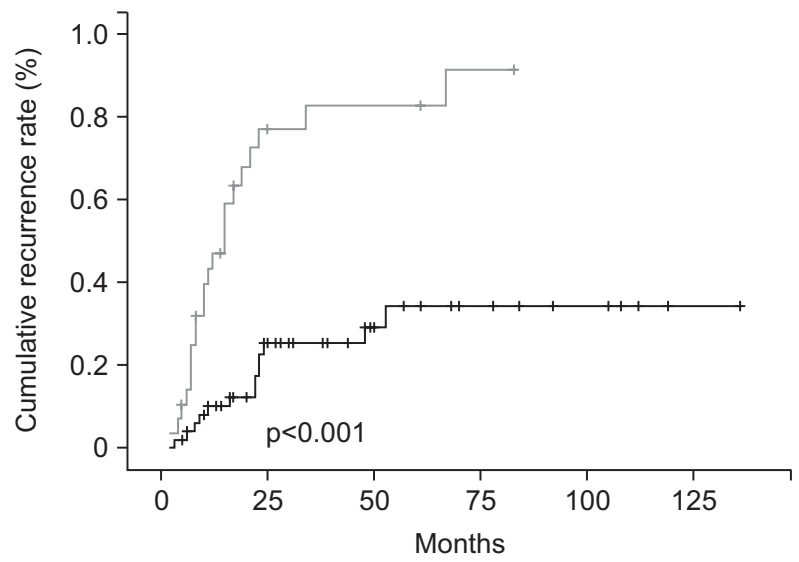

B

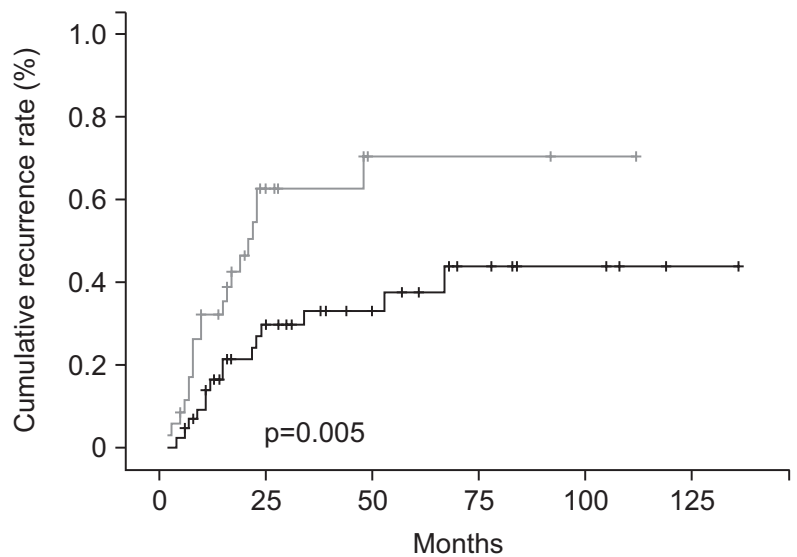

Fig. 2. Cumulative recurrence rates. (A) The recurrence rate of multinodularity (gray line) was increased compared to that of single nodularity (black line) $(75.9 \%$ vs $25 \%, \mathrm{p}<0.001)$. (B) The recurrence rate of an elevated serum $\alpha$-fetoprotein (AFP) ( $>20 \mathrm{ng} / \mathrm{mL}$ ) level (gray line) was increased compared to that of a normal serum AFP ( $\leq 20 \mathrm{ng} / \mathrm{mL}$ ) level (black line) ( $55.6 \%$ vs $33.3 \%, \mathrm{p}=0.005)$.

25\%; $\mathrm{p}<0.001)$. The 1-, 2-, and 3-year cumulative recurrence rates of single nodularity were $9.9 \%, 25.1 \%$, and $25.1 \%$, respectively, and those of multinodularity were $47 \%, 77.1 \%$, and $82.8 \%$, respectively (Fig. 2A). The recurrence rate of patients with elevated serum AFP (>20 ng/mL) level (20/36 patients) was higher than that of with normal serum AFP $(\leq 20 \mathrm{ng} / \mathrm{mL})$ level (15/45 patients) (55.6\% vs 33.3\%; $p=0.005)$. The $1-, 2-$, and 3-year cumulative recurrence rates of patients with normal serum AFP ( $\leq 20 \mathrm{ng} / \mathrm{mL}$ ) level were 16.1\%, 29.6\%, and 33\%, respectively, and those of patients with elevated serum AFP ( $>20$ ng/mL) level were 32\%, 62.8\%, and 62.8\%, respectively (Fig. 2B).

\section{Survival}

The overall 1-, 2-, and 3-year cumulative survival rates of the 287 patients treated with TACE were 75.8\%, 66.5\%, and 52\%, respectively. The survival rate of patients with TACE-induced CR was higher than that of patients without CR ( $<<0.001)$. The cumulative survival rate in patients without or with $\mathrm{CR}$ was $66.4 \%$ versus $96.2 \%$ after 1 year, 58.2\% versus $84.9 \%$ after 2 years, and $45.6 \%$ versus $64.8 \%$ after 3 years.

\section{Predictive factors for survival}

Univariate analysis showed that Child-Turcotte-Pugh (CTP) score B or C (HR, 1.606; 95\% CI, 1.130 to 2.283; p=0.008), elevated serum AFP (>20 ng/mL) level (HR, 2.277; 95\% CI, 1.569 to 3.306; $\mathrm{p}<0.001)$, tumor size ( $>5 \mathrm{~cm})(\mathrm{HR}, 1.770$; 95\% CI, 1.234 to $2.539 ; \mathrm{p}=0.002$ ), multinodularity ( $\mathrm{HR}, 1.747 ; 95 \% \mathrm{CI}, 1.216$ to $2.510 ; \mathrm{p}=0.003$ ) and portal vein thrombosis (HR, 2.893; 95\% CI, 1.872 to $4.470 ; \mathrm{p}<0.001$ ) were correlated with survival after TACE. Multivariate analysis revealed that independent predictor of survival were CTP score B or C (HR, 1.616; 95\% CI, 1.116 to 2.339; $\mathrm{p}=0.011$ ), elevated serum AFP ( $>20 \mathrm{ng} / \mathrm{mL}$ ) level (HR, $1.801 ; 95 \%$ CI, 1.215 to $2.668 ; p=0.003)$, tumor size $(>5 \mathrm{~cm})(\mathrm{HR}$,
$1.618 ; 95 \% \mathrm{CI}, 1.093$ to $2.394 ; \mathrm{p}=0.016)$, multinodularity (HR, 1.468 ; $95 \%$ CI, 1.011 to $2.130 ; p=0.044$ ) and portal vein thrombosis (HR, 2.389; 95\% CI, 1.534 to 3.721; $\mathrm{p}<0.001$ ) (Table 4).

The survival rate of HCC patients with CTP score A was higher than that of HCC patients with CTP score B or C. The overall 1-, 2-, and 3-year cumulative survival rates were lower in patients with CTP score B or C than in patients with CTP score A (65\% vs $81.2 \%$, $58.6 \%$ vs $69.9 \%$, and $41.2 \%$ vs $57.6 \%$, respectively; $p=0.007$ ) (Fig. $3 \mathrm{~A}$ ). The survival rate of HCC patients with normal serum AFP $(\leq 20 \mathrm{ng} / \mathrm{mL})$ level was higher than that of HCC patients with elevated serum AFP (>20 ng/ $\mathrm{mL}$ ) level. The overall 1-, 2-, and 3-year cumulative survival rates were lower in patients with elevated serum AFP (>20 ng/ $\mathrm{mL})$ level than in patients with normal serum AFP $(\leq 20 \mathrm{ng} / \mathrm{mL})$ level (70\% vs $87.5 \%$, $56 \%$ vs $80.1 \%$, and $40.6 \%$ vs $66.2 \%$, respectively; $\mathrm{p}<0.001$ ) (Fig. 3B). The survival rate of HCC patients with tumor size $(\leq 5 \mathrm{~cm})$ was higher than that of HCC patients with tumor size ( $>5 \mathrm{~cm}$ ). The overall 1-, 2-, and 3-year cumulative survival rates were lower in patients with tumor size $(>5$ $\mathrm{cm})$ than in patients with tumor size $(\leq 5 \mathrm{~cm})(62.8 \%$ vs $81.9 \%$, $51.1 \%$ vs $73.2 \%$, and $46.4 \%$ vs $55.1 \%$, respectively; $p=0.002$ ) (Fig. 3C). The survival rate of HCC patients with single nodularity was higher than that of HCC patients with multinodularity. The overall 1-, 2-, and 3-year cumulative survival rates were lower in patients with multinodularity than in patients with single nodularity (70\% vs $83.5 \%, 59.6 \%$ vs $75 \%$, and $43.3 \%$ vs $63.1 \%$, respectively; $p=0.002$ ) (Fig. 3D). The survival rate of HCC patients without portal vein thrombosis was higher than that of HCC patients with portal vein thrombosis. The overall 1-, 2-, and 3-year cumulative survival rates were lower in patients with portal vein thrombosis than in patients without portal vein thrombosis ( $42.4 \%$ vs $81.6 \%, 37.7 \%$ vs $71.3 \%$, and $20.2 \%$ vs $56.8 \%$, respectively; $\mathrm{p}<0.001$ ) (Fig. 3E). 
Table 4. Predictive Factors for Survival

\begin{tabular}{|c|c|c|c|c|c|c|}
\hline \multirow{2}{*}{ Variable } & \multicolumn{3}{|c|}{ Univariate analysis } & \multicolumn{3}{|c|}{ Multivariate analysis } \\
\hline & HR & $95 \% \mathrm{CI}$ & p-value & HR & $95 \% \mathrm{CI}$ & p-value \\
\hline Age (>60 yr) & 1.282 & $0.903-1.819$ & 0.164 & & & \\
\hline Male sex & 1.023 & $0.702-1.492$ & 0.904 & & & \\
\hline $\mathrm{HBV}$ & 1.169 & $0.728-1.877$ & 0.518 & & & \\
\hline $\mathrm{HCV}$ & 1.420 & $0.954-2.113$ & 0.084 & & & \\
\hline Liver cirrhosis & 0.827 & $0.549-1.245$ & 0.362 & & & \\
\hline MELD score (>10) & 1.388 & $0.821-2.345$ & 0.221 & & & \\
\hline CTP score (A/B, C) & 1.606 & $1.130-2.283$ & 0.008 & 1.616 & $1.116-2.339$ & 0.011 \\
\hline AFP (>20 ng/mL) & 2.277 & $1.569-3.306$ & $<0.001$ & 1.801 & $1.215-2.668$ & 0.003 \\
\hline Tumor size (>5 cm) & 1.770 & $1.234-2.539$ & 0.002 & 1.618 & $1.093-2.394$ & 0.016 \\
\hline Nodularity (multinodular) & 1.747 & $1.216-2.510$ & 0.003 & 1.468 & $1.011-2.130$ & 0.044 \\
\hline Portal vein thrombosis & 2.893 & $1.872-4.470$ & $<0.001$ & 2.389 & $1.534-3.721$ & $<0.001$ \\
\hline
\end{tabular}

HR, hazard ratio; CI, confidence interval; HBV, hepatitis B virus; HCV, hepatitis C virus; MELD, model for end-stage liver disease; CTP, ChildTurcotte-Pugh; AFP, $\alpha$-fetoprotein.
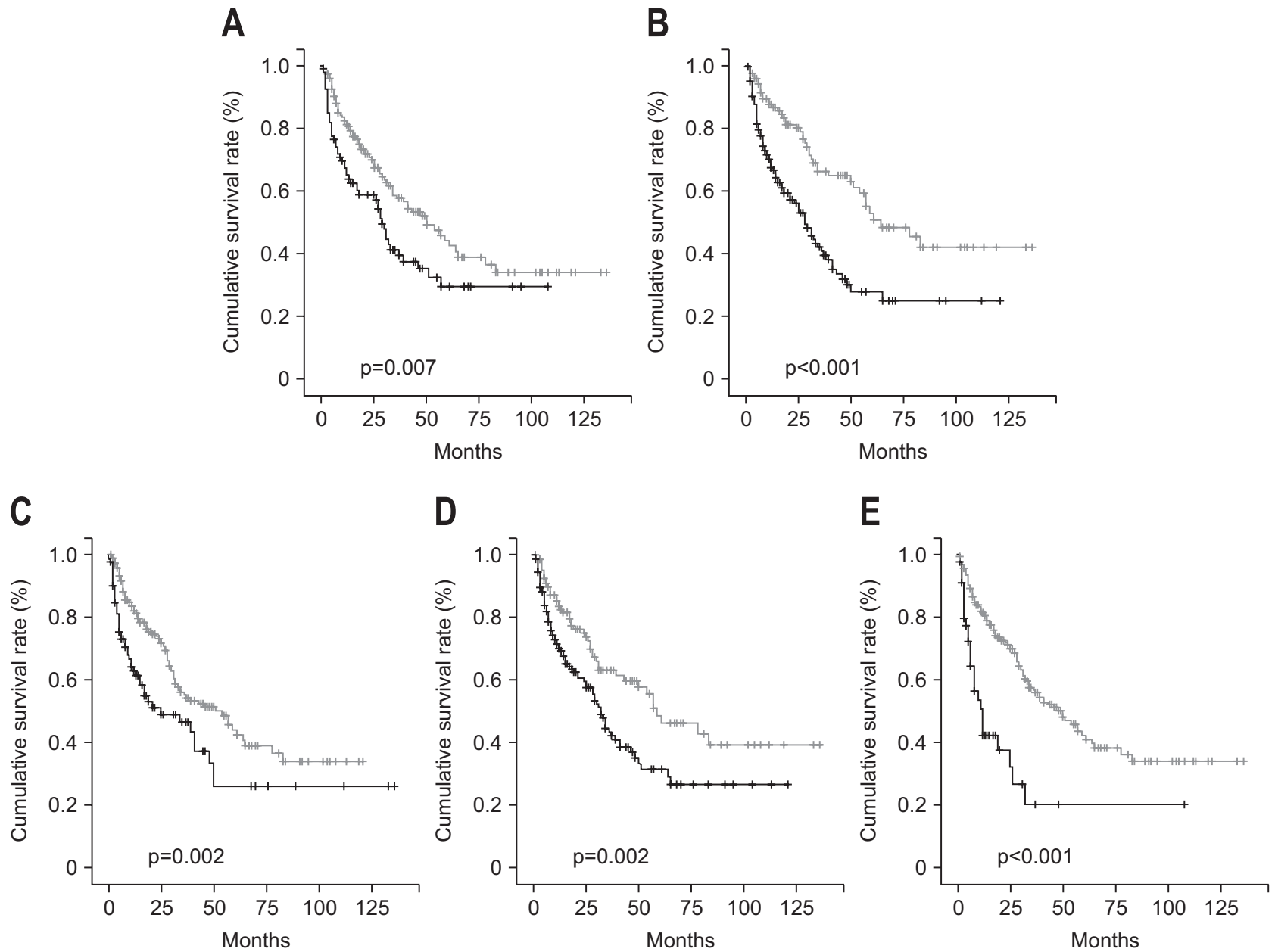

Fig. 3. Cumulative survival rates. (A) The survival rate of hepatocellular carcinoma (HCC) patients with Child-Turcotte-Pugh (CTP) score A (gray line) was increased compared to that of HCC patients with a CTP score of B or C (black line) ( $p=0.007)$. (B) The survival rate of HCC patients with normal serum $\alpha$-fetoprotein (AFP) $(\leq 20 \mathrm{ng} / \mathrm{mL}$ ) levels (gray line) was increased compared to that of HCC patients with elevated serum AFP ( $>20$ $\mathrm{ng} / \mathrm{mL}$ ) levels (black line) $(\mathrm{p}<0.001)$. (C) The survival rate of HCC patients with a tumor size of less than or equal to $5 \mathrm{~cm}$ (gray line) was increased compared to that of HCC patients with tumor size greater than $5 \mathrm{~cm}$ (black line) ( $p=0.002)$. (D) The survival rate of HCC patients with single nodularity (gray line) was increased compared to that of HCC patients with multinodularity (black line) ( $\mathrm{p}=0.002)$. (E) The survival rate of HCC patients without portal vein thrombosis (gray line) was increased compared to that of HCC patients with portal vein thrombosis (black line) ( $<<0.001)$. 


\section{DISCUSSION}

HCC is one of the most common cancers worldwide, and is associated with a high mortality rate. Curative therapies such as hepatic resection, ablation therapy, or liver transplantation are feasible in $20 \%$ to $40 \%$ of patients. TACE is the most commonly used treatment modalities when curative treatment is not indicated. In the present study, we evaluated the predictive factors for CR and recurrence after CR in patients treated with TACE as a first therapy. Single nodularity and tumor size $(\leq 5 \mathrm{~cm})$ were predictive factors for $\mathrm{CR}$, and multinodularity and elevated serum AFP (>20 ng/mL) level were predictive factors for recurrence after CR. Tumor nodularity were significantly associated with both $\mathrm{CR}$ and recurrence after $\mathrm{CR}$. Firstly, in terms of $\mathrm{CR}$, single nodular HCC can be treated effectively with TACE by occluding tumor supplying arteries those are not relatively complex tumor feeding routes comparing with multinodular HCC. In view of tumor size, a strict correlation of $\mathrm{CR}$ and size has previously been pointed out by Ebied et al., ${ }^{22}$ reporting the response to lobal TACE as inversely proportional to tumor size (response in $70 \%$ of HCCs $<3 \mathrm{~cm}$, in $56 \%$ of HCCs 3 to $5 \mathrm{~cm}$, and in 43\% of HCCs >10 cm). Recently, Golfieri et al. ${ }^{23}$ reported that HCCs $\leq 5 \mathrm{~cm}$ were the best responders to superselective TACE; nodules $\leq 2 \mathrm{~cm}$ and nodules between 2.1 and $5 \mathrm{~cm}$ had similar and high CR rates after the first superselective TACE (68\% and 64\%), whereas in HCCs $>5 \mathrm{~cm}$, CR rate significantly decreased (25\%). In this study, CR rate was $87.7 \%$ in $\leq 5 \mathrm{~cm}$ group and $12.3 \%$ in $>5 \mathrm{~cm}$ group. Secondly, multinodularity and elevated serum AFP ( $>20 \mathrm{ng} / \mathrm{mL}$ ) level were predictive factors for recurrence after CR. In respect of multinodularity, it is hypothesized that TACE, as a regional treatment, cannot target undetected satellite lesions outside of the zone of TACE-induced necrosis, such that tumor recurrence is higher in multinodular HCC. Microscopic remnant tumors may not be detectable by imaging studies such as CT during the early phase after TACE. ${ }^{4}$ Lesions that were not detected by CT, but were detected using TACE were reported in $45 \%$ of cases. ${ }^{24}$ Thus, closer surveillance should be considered in patients with multinodular HCC even if they are in remission radiologically after TACE. An elevated serum AFP (>20 ng/mL) level was also associated with recurrence after CR. Generally, HCC with high serum AFP level shows a poorer differentiation grade than does HCC with low serum AFP level. ${ }^{25}$ Our results suggest that HCC associated with elevated serum AFP level needs to be followed more closely considering the additional locoregional treatments if possible. ${ }^{26}$ Serum AFP has roles in both immune system inhibition and promotion of cancer cell growth. Serum AFP may inhibit immune function in the body by altering the ratio of $\mathrm{CD} 4+$ and $\mathrm{CD} 8+\mathrm{T}$ lymphocyte subsets and inducing lymphocyte death. Hypofunction of the immune system is an important factor for susceptibility to recurrence and metastasis. Decreases in serum AFP level after locoregional therapy can predict the tumor response and survival. ${ }^{27}$ Although 10\% to 30\% of patients with HCC are negative for AFP expression, studies have suggested that AFP is significant in predicting HCC recurrence. ${ }^{28}$ Additionally, we analyzed the predictive factors for OS, and CTP score B or C, elevated serum AFP ( $>20 \mathrm{ng} / \mathrm{mL}$ ) level, tumor size ( $>5$ $\mathrm{cm})$, multinodularity, and portal vein thrombosis showed significance. Among these factors, tumor size ( $>5 \mathrm{~cm})$, multinodularity, and elevated serum AFP ( $>20 \mathrm{ng} / \mathrm{mL}$ ) level were the predictive factors for $\mathrm{CR}$ and recurrence after $\mathrm{CR}$. Threrefore, this result reflects that the CR and recurrence after CR of first TACE have an important bearing on OS.

TACE as a curative treatment is based on the recent development of subsegmental (or superselective) TACE compared with palliative treatment. In subsegmental (or superselective) TACE, a microcatheter is supraselectively inserted into the tumoral feeding artery leaving at least $1 \mathrm{~cm}$ of peritumoral safety margin of liver parenchyma. Anticancer drug is usually dissolved in nonionic contrast medium, and then mixed with lipiodol by pumping through a three way stopcock to make a water in oil emulsion that works as the drug carrier. The total amount of lipiodol used is almost equal to the tumor diameter. The gelatin sponge sheet is cut into cubes approximately $0.5 \mathrm{~mm}$ in diameter. A mixture of lipiodol and anticancer drugs is injected gradually until visualization of the surrounding portal vein or slowed arterial flow is seen. Subsequently, gelatin sponge particles are injected until arterial flow is stopped. In the case of multiple HCC nodules, superselective TACE is performed for each nodule. ${ }^{29}$

Our study has limitations in that these data were obtained from a single center and a retrospective study. Secondly, although TACE should be used in patients with HCC diagnosed at an intermediate stage in the BCLC staging system, we included the patients with early-stage HCC (stages I and II) who were not suitable for resection, RFA or liver transplantation because of poor liver function or a location difficult to access by surgery or RFA, or difficulty obtaining a donor liver. The conclusion of this study can give misunderstanding for decision of the treatment strategy. Thirdly, 4 weeks can be too short to determine whether patients achieved CR and it is too difficult to detect residual viable tumor in the remained tumor mass because nonspecific lipiodol accumulation is still present around the tumor and washout of lipiodol cannot be evaluated by the first time CT at 4 weeks after TACE. However, this study identified predictive factors for CR and recurrence after CR in patients with HCC treated with TACE and showed that these factors affect OS.

In conclusion, tumor size $(\leq 5 \mathrm{~cm})$ and single nodularity were predictive factors for $\mathrm{CR}$, and multinodularity and elevated serum AFP (>20 ng/mL) level were predictive factors for recurrence after CR in patients treated with TACE as a first therapy. These factors also had an effect on OS.

\section{CONFLICTS OF INTEREST}

No potential conflict of interest relevant to this article was reported. 


\section{ACKNOWLEDGEMENTS}

This study was supported by the Soonchunhyang University Research Fund.

\section{REFERENCES}

1. Ferlay J, Shin HR, Bray F, Forman D, Mathers C, Parkin DM. Estimates of worldwide burden of cancer in 2008: GLOBOCAN 2008. Int J Cancer 2010;127:2893-2917.

2. Montalto G, Cervello M, Giannitrapani L, Dantona F, Terranova A, Castagnetta LA. Epidemiology, risk factors, and natural history of hepatocellular carcinoma. Ann N Y Acad Sci 2002;963:13-20.

3. Scartozzi M, Faloppi L, Bianconi M, et al. The role of LDH serum levels in predicting global outcome in HCC patients undergoing TACE: implications for clinical management. PLoS One 2012;7:e32653.

4. Park W, Chung YH, Kim JA, et al. Recurrences of hepatocellular carcinoma following complete remission by transarterial chemoembolization or radiofrequency therapy: focused on the recurrence patterns. Hepatol Res 2013;43:1304-1312.

5. Sugioka A, Tsuzuki T, Kanai T. Postresection prognosis of patients with hepatocellular carcinoma. Surgery 1993;113:612-618.

6. El-Serag HB, Marrero JA, Rudolph L, Reddy KR. Diagnosis and treatment of hepatocellular carcinoma. Gastroenterology 2008;134:1752-1763.

7. Forner A, Hessheimer AJ, Isabel Real M, Bruix J. Treatment of hepatocellular carcinoma. Crit Rev Oncol Hematol 2006;60:89-98.

8. Yu SJ. A concise review of updated guidelines regarding the management of hepatocellular carcinoma around the world: 20102016. Clin Mol Hepatol 2016;22:7-17.

9. Kohles N, Nagel D, Jüngst D, Durner J, Stieber P, Holdenrieder S. Prognostic relevance of oncological serum biomarkers in liver cancer patients undergoing transarterial chemoembolization therapy. Tumour Biol 2012;33:33-40.

10. Takayasu K, Arii S, Ikai I, et al. Prospective cohort study of transarterial chemoembolization for unresectable hepatocellular carcinoma in 8510 patients. Gastroenterology 2006;131:461-469.

11. Cabibbo G, Genco C, Di Marco V, et al. Predicting survival in patients with hepatocellular carcinoma treated by transarterial chemoembolisation. Aliment Pharmacol Ther 2011;34:196-204.

12. Hsu KF, Chu CH, Chan DC, et al. Superselective transarterial chemoembolization vs hepatic resection for resectable early-stage hepatocellular carcinoma in patients with Child-Pugh class a liver function. Eur J Radiol 2012;81:466-471.

13. Tateishi R, Shiina S, Yoshida H, et al. Prediction of recurrence of hepatocellular carcinoma after curative ablation using three tumor markers. Hepatology 2006;44:1518-1527.

14. Izumi N, Asahina Y, Noguchi 0, et al. Risk factors for distant recurrence of hepatocellular carcinoma in the liver after complete coagulation by microwave or radiofrequency ablation. Cancer 2001;91:949-956.
15. Imamura H, Matsuyama $Y$, Tanaka E, et al. Risk factors contributing to early and late phase intrahepatic recurrence of hepatocellular carcinoma after hepatectomy. J Hepatol 2003;38:200-207.

16. Nouso K, Ito Y, Kuwaki K, et al. Prognostic factors and treatment effects for hepatocellular carcinoma in Child C cirrhosis. Br J Cancer 2008;98:1161-1165.

17. Arimura E, Kotoh K, Nakamuta M, Morizono S, Enjoji M, Nawata H. Local recurrence is an important prognostic factor of hepatocellular carcinoma. World J Gastroenterol 2005;11:5601-5606.

18. Ueno S, Tanabe G, Nuruki K, et al. Prognosis of hepatocellular carcinoma associated with Child class B and C cirrhosis in relation to treatment: a multivariate analysis of 411 patients at a single center. J Hepatobiliary Pancreat Surg 2002;9:469-477.

19. Bruix J, Sherman M; American Association for the Study of Liver Diseases. Management of hepatocellular carcinoma: an update. Hepatology 2011;53:1020-1022.

20. Ueno S, Tanabe G, Nuruki K, et al. Prognostic performance of the new classification of primary liver cancer of Japan (4th edition) for patients with hepatocellular carcinoma: a validation analysis. Hepatol Res 2002;24:395-403.

21. Lencioni R, Llovet JM. Modified RECIST (mRECIST) assessment for hepatocellular carcinoma. Semin Liver Dis 2010;30:52-60.

22. Ebied OM, Federle MP, Carr BI, et al. Evaluation of responses to chemoembolization in patients with unresectable hepatocellular carcinoma. Cancer 2003;97:1042-1050.

23. Golfieri R, Renzulli M, Mosconi C, et al. Hepatocellular carcinoma responding to superselective transarterial chemoembolization: an issue of nodule dimension? J Vasc Interv Radiol 2013;24:509-517.

24. Rou WS, Lee BS, Moon HS, Lee ES, Kim SH, Lee HY. Risk factors and therapeutic results of early local recurrence after transcatheter arterial chemoembolization. World J Gastroenterol 2014;20:69957004.

25. Okuda H, Nakanishi T, Takatsu K, et al. Comparison of clinicopathological features of patients with hepatocellular carcinoma seropositive for alpha-fetoprotein alone and those seropositive for des-gamma-carboxy prothrombin alone. J Gastroenterol Hepatol 2001;16:1290-1296.

26. Veltri A, Moretto P, Doriguzzi A, Pagano E, Carrara G, Gandini G. Radiofrequency thermal ablation (RFA) after transarterial chemoembolization (TACE) as a combined therapy for unresectable nonearly hepatocellular carcinoma (HCC). Eur Radiol 2006;16:661669.

27. Riaz A, Ryu RK, Kulik LM, et al. Alpha-fetoprotein response after locoregional therapy for hepatocellular carcinoma: oncologic marker of radiologic response, progression, and survival. J Clin Oncol 2009;27:5734-5742.

28. Evdokimova VN, Liu Y, Potter DM, Butterfield LH. AFP-specific CD4+ helper T-cell responses in healthy donors and HCC patients. J Immunother 2007;30:425-437.

29. Matsui 0. Current status of hepatocellular carcinoma treatment in Japan: transarterial chemoembolization. Clin Drug Investig 2012;32 Suppl 2:3-13. 